\title{
Clinical impact of defibrillation testing at the time of implantable cardioverter-defibrillator insertion
}

\author{
Claudio Hadid $^{1}$, Felipe Atienza ${ }^{1}$, Boris Strasberg ${ }^{2}$, Ángel Arenal ${ }^{1}$, \\ Pablo Codner ${ }^{2}$, Esteban González-Torrecilla ${ }^{1}$, Tomás Datino ${ }^{1}$, \\ Tamara Percal ${ }^{1}$, Jesús Almendral ${ }^{1}$, Mercedes Ortiz ${ }^{1}$, Raphael Martins ${ }^{3}$, \\ Nieves Martinez-Alzamora ${ }^{4}$, Francisco Fernandez Aviles ${ }^{1}$ \\ ${ }^{1}$ Hospital General Universitario Gregorio Marañón, Madrid, Spain \\ ${ }^{2}$ Rabin Medical Center, Tel Aviv University, Tel Aviv, Israel \\ ${ }^{3} \mathrm{CHU}$ Nancy, France \\ ${ }^{4}$ Universidad Politécnica de Valencia, Valencia, Spain
}

\begin{abstract}
Background: Ventricular fibrillation is routinely induced during implantable cardioverter-defibrillator insertion to assess defibrillator performance, but this strategy is experiencing a progressive decline. We aimed to assess the efficacy of defibrillator therapies and long-term outcome in a cohort of patients that underwent defibrillator implantation with and without defibrillation testing.
\end{abstract}

Methods: Retrospective observational series of consecutive patients undergoing initial defibrillator insertion or generator replacement. We registered spontaneous ventricular arrhythmias incidence and therapy efficacy, and mortality.

Results: A total of 545 patients underwent defibrillator implantation (111 with and 434 without defibrillation testing). After 19 (range 9-31) months of follow-up, the death rate per observation year (4\% vs. 4\%; $p=0.91$ ) and the rate of patients with defibrillator-treated ventricular arrhythmic events per observation year (with test: $10 \%$ vs. without test: $12 \% ; p=0.46$ ) were similar. The generalized estimating equations-adjusted first shock probability of success in patients with test (95\%; CI 88-100\%) vs. without test (98\%; CI 96-100\%; $p=0.42$ ) and the proportion of successful antitachycardia therapies (with test: $87 \%$ vs. without test: $80 \%$; $p=0.35$ ) were similar between groups. There was no difference in the annualized rate of failed first shock per patient and per shocked patient between groups (5\% vs. 4\%; $p=0.94)$.

Conclusions: In this observational study, that included an unselected population of patients with a defibrillator, no difference was found in overall mortality, first shock efficacy and rate of failed shocks regardless of whether defibrillation testing was performed or not. (Cardiol J 2015; 22, 3: 253-259)

Key words: implantable cardioverter-defibrillator, defibrillation testing, ventricular fibrillation, defibrillator shocks, mortality

Address for correspondence: Felipe Atienza, MD, PhD, Cardiology Department, Hospital General Universitario Gregorio Marañón, C/Dr Esquerdo, 46, 28007 Madrid, Spain, tel: +34915868281, e-mail: fatienzaf@secardiologia.es 


\section{Introduction}

The assessment of defibrillation efficacy has long been the standard of care during implantable cardioverter-defibrillator (ICD) insertion, and is recommended by ICDs manufacturers and scientific guidelines $[1,2]$. However, this strategy is currently being questioned due to the high probability of current devices to successfully defibrillate ventricular arrhythmias, the low incidence of spontaneous ventricular fibrillation (VF) $(\sim 10 \%)$ and the high efficacy of dedicated antitachycardia pacing (ATP) strategies [2-8]. Moreover, defibrillation testing (DT) is not without a risk, and complications associated to VF induction, shock delivery and anesthesia may occur [2, 3, 9-12]. For all these reasons, the proportion of patients undergoing any type of DT, especially among primary prevention ICD implantation, is experiencing a progressive decline in routine clinical practice [12-15]. The prospective, non-randomized SAFE-ICD study selected centers according to their common practice of performing or not performing DT at implantation and found no different outcome between groups [8]. However, that study only included de-novo implants and did not report data on ATP therapy.

The purpose of the present study was to assess the efficacy of ICD therapies (both shocks and ATP) and long-term clinical outcome in a cohort of consecutive patients, including de-novo implants and generator replacements, that underwent ICD implantation with $(+)$ and without (-) DT at the time of ICD insertion.

\section{Methods}

We retrospectively reviewed the records of a series of consecutive patients $>18$ years old, in whom an ICD had been implanted in two tertiary-care teaching hospitals (Hospital General Universitario Gregorio Marañón and Rabin Medical Center) during a period of 3 years, following the implementation of a non-DT policy at our institutions. DT was recommended in right pectoral ICD implantation, $\mathrm{R}$ wave signal $<5 \mathrm{mV}$ or not able to be measured due to pacing, abandoned leads, implanted electronic devices, chronic treatment with amiodarone and patients with hypertrophic cardiomyopathy or channelopathies. However, the final decision to test was left at the discretion of the operator. In case any type of DT was considered necessary defibrillation safety margin estimation was routinely performed [16]. In the DT + group, VF was induced by standard T-wave shock or a $50-\mathrm{Hz}$ burst. A protocol of 2 defibrillations at $10 \mathrm{~J}$ below the maximum output of the device was applied with a 5-min rest period between VF inductions. If the first shock failed, but the second was successful, the patient was also considered to have adequate DT. If the defibrillation attempt was unsuccessful certain strategies were adopted to overcome this situation (i.e. right ventricular [RV] lead repositioning) and the protocol was repeated. All patients signed written informed consent. All spontaneous tachyarrhythmic episodes with ICD stored electrograms that resulted in ventricular therapies were reviewed by two independent observers (C.H. and P.C.), to assess efficacy of the delivered therapy. Disagreements were resolved by consensus with a third observer.

All ICD implantations were performed at the electrophysiology laboratory under local anesthesia and conscious sedation. Only pectoral implantations with dual-coil shocking leads placed in the RV apex were included in the study. Patients received high-output $(\geq 35 \mathrm{~J}$ ) or standard active can devices manufactured by Medtronic (Minneapolis, MN, USA), Boston Scientific (St. Paul, MN, USA) or Biotronik (Berlin, Germany). Device programming was performed according to patient clinical status and left at the discretion of the responsible physician, but all shocks were programmed at the highest available output of the device. However, a standardized ATP programming in the fast ventricular tachycardia (VT) zone was recommended in all patients [17]. Patients were first followed 3 months after device implantation and every 6 months thereafter.

\section{Statistical analysis}

We used an approximate normal test for the equality of rates for sample size estimation, where a total of 111 patients in the DT + group and 434 in the DT-group would be able to detect an absolute error in mortality rates of 0.06 , with $80 \%$ statistical power and a significance level of $5 \%$. Continuous variables were assessed for normality using the Wilk-Shapiro test. Normally distributed variables are expressed as mean \pm standard deviation, whereas those with nonparametric distribution are presented as median and interquartile range (IQR). Categorical variables are reported as number and percentage. Comparisons of normal continuous baseline characteristics were made using Student's t-test. In case of non-normally distributed continuous variables, nonparametric tests were used. Comparisons of categorical variables were done using the Fisher's exact test or $\chi^{2}$ test, as appropriate. 
Table 1. Patients' baseline characteristics.

\begin{tabular}{lccc}
\hline Variable & DT $+(\mathbf{n}=111)$ & DT- $(\mathbf{n}=434)$ & P \\
\hline Age [years] & $67(54-74)$ & $68(59-76)$ & $0.03^{*}$ \\
Male & $95(86 \%)$ & $360(83 \%)$ & $0.52^{* *}$ \\
Heart disease etiology: & & $0.12^{* *}$ \\
Ischemic & $80(72 \%)$ & $279(64 \%)$ & $0.08^{* *}$ \\
Dilated cardiomyopathy & $12(11 \%)$ & $77(18 \%)$ & $0.20^{* *}$ \\
Channelopathies & $5(4.5 \%)$ & $10(2.3 \%)$ & $0.88^{* *}$ \\
Hypertrophic cardiomyopathy & $6(5.4 \%)$ & $22(5 \%)$ & $0.28^{* *}$ \\
Other & $8(7 \%)$ & $46(10 \%)$ & $0.16^{* *}$ \\
Ejection fraction $\leq 0.35$ & $69 / 111(62 \%)$ & $300 / 413(73 \%)$ & $0.08^{* *}$ \\
Indication secondary prevention & $69(62 \%)$ & $227(52 \%)$ & $0.22^{* *}$ \\
De novo ICD implantation & $95(86 \%)$ & $349(80 \%)$ & $<0.01^{* *}$ \\
Type of ICD: & & & $N^{* * *}$ \\
Single chamber & $72(65 \%)$ & $270(62 \%)$ & $0.01^{* * *}$ \\
Dual chamber & $21(19 \%)$ & $44(10 \%)$ & $0.01^{* * *}$ \\
CRT-ICD & $18(16 \%)$ & $120(28 \%)$ & $0.01^{* *}$ \\
Antiarrhythmic drug therapy (amiodarone) & $38(34 \%)$ & $95(22 \%)$ & \\
\hline
\end{tabular}

Continuous variables are expressed as median and interquartile range; *Mann-Whitney U test. Sig 2-sided; **Pearson's $\chi^{2}$ test. Sig 2-sided; ***Approximate normal test for adjusted residuals. Sig. 2-sided; CRT-ICD — cardiac resynchronization therapy with defibrillation capabilities; DT — defibrillation testing; ICD — implantable cardioverter-defibrillator; NS — not significant

Incidence rates of death and arrhythmic events between the cohorts of DT + and DT- patients were compared using an approximate normal test for the equality of rates. The annualized rate for each arrhythmic episode type per patient month was calculated by dividing the total number of episodes by type by months of follow-up for each patient group (DT+ vs. DT-), where the weight assigned to each patient is the proportion of overall follow-up that the patient contributed to the aggregate follow-up [7]. Annualized rates of events per patient in both cohorts were compared using the Generalized Linear Model (GLZM) and the Wald $\chi^{2}$ test. Generalized estimating equations (GEE) method was used to compare the efficacy of ICD therapies in the two cohorts, adjusting for multiple episodes per patient [18]. Kaplan-Meier survival curves and log-rank test were used to compare survival between both groups. For statistical analysis we used SPSS version 19.0 (SPSS Inc.; Chicago, IL). All probability (p) values were 2 -sided and statistical significance was established at $\mathrm{p}<0.05$.

\section{Results}

We included 548 consecutive patients that represent the total number of ICD implants performed at the participating centers during the inclusion period at each site, including generator replacements. Patients were followed for a median of 19 months (IQR 9-31). Three $(0.68 \%)$ patients could not be contacted and were considered lost to follow-up and excluded from analysis. Table 1 shows the clinical characteristics of patients included in the study. In 111 (20\%) patients, DT was performed at the time of ICD implantation. DT + patients were slightly younger, less likely to receive a cardiac resynchronization therapy with defibrillation capabilities (CRT-ICD) and more likely to be treated with antiarrhythmic drugs and to receive a dual-chamber device. Other baseline clinical characteristics were comparable between groups.

\section{Patients' clinical outcomes}

Patients in the DT + group were followed for a longer period than DT- patients (32 [18-45] vs. 15 [9-26] months; $\mathrm{p}<0.001$ ). After adjusting the event rate by the follow-up duration, there was no significant difference in the death rate per observation year (DT+: 0.04 vs. DT-: 0.04; $\mathrm{p}=0.91$ ) nor in the rate of patients with ventricular arrhythmic events per observation year (DT+: 0.10 vs. DT-: $0.12 ; \mathrm{p}=0.46$ ) (Table 2). Similarly, there was no difference in the rate of patients with VF per observation year and only a trend towards a slightly higher rate of patients with VT per observation year in the DT-group. 
Table 2. Patients' clinical outcomes during follow-up.

\begin{tabular}{lccc}
\hline Incidence rate (patient-year observation) & DT+ $(\mathbf{n}=\mathbf{1 1})$ & DT- $\mathbf{n}=\mathbf{4 3 4})$ & $\mathbf{P}$ \\
\hline Death & 0.04 & 0.04 & 0.91 \\
Patients with ventricular arrhythmic events: & 0.10 & 0.12 & 0.46 \\
Patients with ventricular fibrillation & 0.08 & 0.05 & 0.16 \\
Patients with ventricular tachycardia & 0.05 & 0.09 & 0.07 \\
Patients with therapies: & 0.10 & 0.12 & 0.46 \\
Shock & 0.08 & 0.07 & 0.69 \\
Antitachycardia pacing & 0.05 & 0.07 & 0.16 \\
\hline
\end{tabular}

Approximate normal test for equality of rates. Sig. 2-sided; DT — defibrillation testing

Table 3. Efficacy of appropriate therapies for ventricular arrhythmias.

\begin{tabular}{lccc}
\hline & $\begin{array}{c}\text { DT }+ \\
\text { (n }=\mathbf{3 1})\end{array}$ & $\begin{array}{c}\text { DT- } \\
\text { (n = 78) }\end{array}$ & P \\
\hline Successful shocks & 38 & 136 & \\
Failed shocks & 2 & 3 & \\
Successful shocks/ & $95 \%$ & $98 \%$ & 0.42 \\
/shocks \pm & 79 & 196 & \\
Successful ATP & 46 & 133 & \\
Failed ATP & $87 \%$ & $80 \%$ & 0.35 \\
Successful ATP/ATP \pm & & & \\
\hline
\end{tabular}

\pm Generalized estimating equations adjustment to account for multiple episodes; ATP — antitachycardia pacing; DT — defibrillation testing

\section{ICD therapy frequency and efficacy by DT group at implant}

During the follow-up period, 109 (20\%) patients experienced 449 ventricular arrhythmic episodes that required ICD therapy. The absolute proportion of shocked patients was higher in the DT + group (22\% vs. $11 \%$; p < 0.01). However, after adjusting for the follow-up duration, there was no difference in the annualized rate of patients receiving shocks or ATP therapies, between DT + vs. DT-groups (Table 2).

As shown in Table 3, there was no difference in the GEE-adjusted first shock probability of success in patients with DT + (95\%; CI 88-100\%) vs. DT(98\%; CI 96-100\%; $\mathrm{p}=0.42$ ). The proportion of successful ATP therapies was greater in the DT+ group, but the difference was not significant (DT+: $87 \%$ vs. DT-: $73 \%$; $\mathrm{p}=\mathrm{NS}$ ).

Five patients had a failed appropriate first shock: 2 patients in the DT + group and 3 in the DT - group (Table 4). This accounts for $2.8 \%(5 / 179)$ of total appropriate ICD shocks delivered in the study and $0.9 \%(5 / 545)$ of patients followed in the study. After adjusting for the differences in follow-up duration, there was no difference between groups in the annualized rate of failed first shock per patient (DT+: 0.01 vs. DT-: $<0.01 ; \mathrm{p}=0.71)$ and per shocked patient (DT+: 0.05 vs. DT-: $0.04 ; \mathrm{p}=0.94$ ). Table 4 shows the clinical characteristics of patients with a failed first shock. In all cases, the second shock successfully terminated the ventricular arrhythmia.

\section{Mortality}

Forty $(7.3 \%)$ patients died during the follow-up: 13 in the DT+ group (6 non-sudden cardiac

Table 4. Clinical characteristics of patients with a failed first ICD shock.

\begin{tabular}{lccccccccccc}
\hline Case & Gender & Age & Etiology & EF & Device & $\begin{array}{c}\text { Indication: } \\
\text { Prevention }\end{array}$ & DT & Event & $\begin{array}{c}\text { Zone } \\
\text { AAD }\end{array}$ & $\begin{array}{c}2^{\text {nd }} \text { ICD } \\
\text { shock }\end{array}$ \\
\hline 1 & Male & 70 & Non-ischemic & 0.15 & CRT-ICD & Primary & - & M-VT & VF & Yes & Successful \\
2 & Male & 61 & Ischemic & 0.30 & SC-ICD & Secondary & - & M-VT & VF & No & Successful \\
3 & Male & 60 & Non-ischemic & 0.20 & SC-ICD & Secondary & - & P-VT & VF & No & Successful \\
4 & Male & 59 & Ischemic & 0.30 & DC-ICD & Secondary & + & VF & VF & Yes & Successful \\
5 & Male & 65 & Ischemic & 0.25 & SC-ICD & Secondary & + & VF & VF & Yes & Successful \\
\hline
\end{tabular}

ICD - implantable cardioverter-defibrillator; EF — ejection fraction; Zone — ICD detection zone; AAD — antiarrhythmic drug therapy; SC-ICD - single-chamber ICD; DC-ICD — dual-chamber ICD; M-VT — monomorphic ventricular tachycardia; P-VT — polymorphic ventricular tachycardia; VF — ventricular fibrillation 


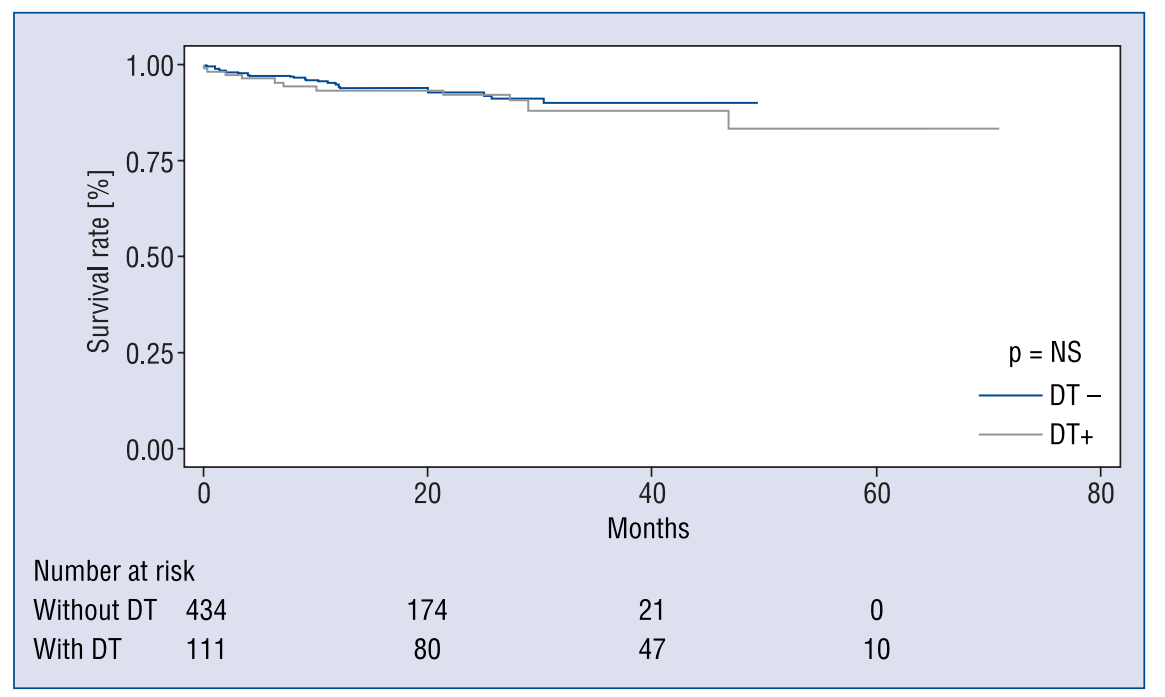

Figure 1. Kaplan-Meier estimates of survival in patients with (DT+) and without (DT-) defibrillation testing.

deaths; 4 non-cardiac deaths; 2 sudden deaths; 1 unknown cause) and 27 in the DT-group (11 non-sudden cardiac death; 11 non-cardiac death; 2 electrical storm; 3 unknown causes). Patients dying due to electrical storm were admitted to the hospital with incessant ventricular arrhythmia that was treated with multiple appropriate ICD discharges that terminated the episodes with the first shock in all instances, while the ICD was working properly. One sudden death in the DT + group occurred in 1 patient with hypertrophic cardiomyopathy. Unknown deaths were non-sudden and no shock delivery occurred. There were no deaths related to device failure or malfunction.

Unadjusted mortality rates were higher in patients with $(12 \%)$ vs. without DT $(6.2 \%$; p = $=0.048$ ). However, after adjusting for the differences in follow-up duration, there was no difference in the annualized rate of death in DT + vs. DT- patients (Table 2). As shown in Figure 1, there were no significant differences in the observed survival rates between DT + vs. DT- patients $(\mathrm{p}=0.9)$.

\section{Discussion}

This observational study in an unselected population of consecutive patients undergoing ICD implantation found similar shock efficacy and long-term clinical outcome regardless of whether DT was performed or not. Therefore, in our study, performing DT did not predict ICD performance and had no clinical impact during the follow-up. These results are representative of the overall population of patients currently undergoing all types of ICD implants at two tertiary referral centers, for all kinds of ICD indications, including ICD with CRT capabilities and generator replacements. Finally, these results are representative of the clinical outcomes of the transition from the traditional DT strategy to the non-DT strategy for most of the patients.

\section{Clinical impact of DT}

ICD implantation is indicated to reduce allcause mortality in patients at risk. In the present study, we observed no differences in the overall mortality rates irrespective of whether DT was performed or not. All-cause mortality rates were similar in the RAFT pilot trial [19], but lower than lower in others observational or retrospective studies [8, 20, 21]. Differences in patient characteristics and follow-up duration might account for these apparent discrepancies. Nevertheless, none of these studies were powered to detected differences in all-cause mortality and sudden cardiac death. On the other hand, in agreement with prior studies, there was no difference between groups in the annualized rate of patients with ventricular arrhythmic episodes and the annualized rate of patients receiving appropriate shocks (8\% vs. 9\%) [19-21].

\section{ICD therapy efficacy in DT+ vs. DT- patients}

The efficacy of ICD to successfully defibrillate induced VF at implant is considered an adequate surrogate of ICD efficacy during follow-up [22]. In the present study, first shock efficacy was $97 \%$ and 
only 5 episodes required a second shock to successfully terminate the arrhythmia, with no differences between DT + (95\%) vs. DT- (98\%) groups. This comparison cannot be assessed statistically due to the small number of unsuccessful shocks. This rate of first shock failure is in agreement to that reported for spontaneous VF in patients with DT $(80-91 \%)$ or safety margin testing $(91 \%)$ at ICD implantation $[2,4,5]$. Similarly, a significant proportion (80-87\%) of VT/fast VT episodes were successfully ATP-terminated without shock delivery, regardless of whether DT was performed. This is consistent with previous studies showing that dedicated ATP programming reduce the need for shocks to terminate VT/VF in a substantial number of patients [7, 17, 23]. In addition, newer devices are able to deliver ATP during energy charge when treating a VF episode, in an attempt to terminate a fast VT detected in the VF zone [24, 25]. Interestingly, we observed no first shock failures following ineffective ATP therapies, both in DT + and DTpatients, since all first shocks were programmed at maximum energy [23].

\section{Prior studies}

Prior retrospective $[5,20,21]$, prospective observational [8] and small randomized [19] studies have reported on the long-term clinical outcome of non-tested vs. patients undergoing DT, mostly in mixed population of primary prevention and CRT-D patients. Despite differences in design, patients' characteristics and follow-up duration, our results are in agreement with most of these studies. Pires and Johnson [5] reported a retrospective analysis of ICD patients assigned to intraoperative DT, limited defibrillation safety margin testing or no DT in a non-randomized fashion, and found no difference in shocks efficacy and sudden death rate, but a higher overall mortality in the non-tested group. Bianchi et al. [21] retrospectively compared the clinical outcome of ICD recipients for primary prevention from centers that routinely performed DT to that of patients in whom no DT was performed, and found no difference in total mortality, cardiovascular mortality or sudden death rates between those groups. Similarly, no differences in survival free of heart transplantation or shock efficacy were found in a retrospective study of CRT-D patients [20]. A small pilot sub-study of the RAFT trial, compared patients with and without DT in a randomized fashion and showed similar ICD therapy efficacy but a non-significant 2 -fold increased all-cause mortality in patients undergoing DT [19]. In the largest study reported to date, the SAFE-ICD study prospectively followed 2,120 patients from centers selected according to their standard implantation practice and found a similar long-term outcome in patients with DT vs. without DT during de novo implants, in terms perioperative complications, ICD therapy efficacy and mortality [8]. Despite the smaller sample size, our study agrees with the SAFE-ICD in their prospective, non-randomized nature, and the lack of outcome differences between groups. However, in our study, no patients were excluded during the recruitment period and all types of ICD implants were considered, including generator replacements. Thus, our study confirms and further expands the SAFE-ICD study results in patients that most likely represent an unselected population currently undergoing ICD implantation with regards to age, gender, indication and device type $[14,16]$. Moreover, this is the first study to provide detailed analyses of the outcome of ICD therapies, both ATP and shocks, showing a similar first shock efficacy in patients following failed ATP therapy in patients with and without DT at implant. Since ATP therapy failure delays time to shock and prolongs VF duration, a lower shock efficacy could have been expected in this context [23, 26]. It is reassuring to note that in our study, all VT/VF episodes following failed ATP were successfully converted when first shock was programmed at maximum energy, regardless DT was performed or not.

\section{Limitations of the study}

Our study has several potential limitations. This is a non-randomized retrospective study with different follow-up times between groups that were adjusted by calculating annualized event rates. Selection bias towards including patients with lower DT thresholds in the non-tested group cannot be excluded. Non-tested patients had lower ejection fraction and this difference may explain the higher rate of CRT implantation in this group. Second, as only dual-coil ICD leads were implanted, our findings should not be extrapolated to patients with single-coil leads. Third, ICD programming was not guided by the result of DT in the tested group and first shocks were set at maximum energy in both groups. The remaining ICD parameters settings were left at the physicians' discretion and were not available for analyses. Nevertheless, the recommended ATP programming strategy was able to successfully terminate a high proportion of treated episodes in previous studies [7, 17, 26]. Finally, 3 patients died for unknown causes, but witnesses' reports reasonably excluded a sudden cause of death. 


\section{Conclusions}

In this observational study, that included an unselected population of consecutive patients undergoing ICD implantation, performing DT at the time of ICD insertion was not associated with improved survival, higher first shock efficacy probability or lower rate of failed ICD shocks as compared to the strategy of withholding DT. Until the results of randomized clinical trials become available [22], the present study questions the need for systematically performing DT in the majority of patients undergoing ICD implantation.

Conflict of interest: Dr Atienza and Prof. Strasberg are on the European Avisory Board of Medtronic. The rest of the authors have no conflicts of interests to report.

\section{References}

1. Curtis AB, Ellenbogen KA, Hammill SC et al. Cinical competency statement: Training pathways for implantation of cardioverter defibrillators and cardiac resynchronization devices. Heart Rhythm, 2004; 1: 371-375.

2. Swerdlow CD, Russo AM, Degroot PJ. The dilemma of ICD implant testing. Pacing Clin Electrophysiol, 2007; 30: 675-700.

3. Viskin S, Rosso R. The top 10 reasons to avoid defibrillation threshold testing during ICD implantation. Heart Rhythm, 2008; 5: 391-393.

4. Blatt JA, Poole JE, Johnson GW et al. No benefit from defibrillation threshold testing in the SCD-HeFT (Sudden Cardiac Death in Heart Failure Trial). J Am Coll Cardiol, 2008; 52: 551-556.

5. Pires LA, Johnson KM. Intraoperative testing of the implantable cardioverter-defibrillator: How much is enough? J Cardiovasc Electrophysiol, 2006; 17: 140-145.

6. Russo AM, Sauer W, Gerstenfeld EP et al. Defibrillation threshold testing: Is it really necessary at the time of implantable cardioverter-defibrillator insertion? Heart Rhythm, 2005; 2: 456-461.

7. Sweeney MO, Sherfesee L, DeGroot PJ, Wathen MS, Wilkoff BL. Differences in effects of electrical therapy type for ventricular arrhythmias on mortality in implantable cardioverter-defibrillator patients. Heart Rhythm, 2010; 7: 353-360.

8. Brignole M, Occhetta E, Bongiorni MG et al. Clinical evaluation of defibrillation testing in an unselected population of 2,120 consecutive patients undergoing first implantable cardioverterdefibrillator implant. J Am Coll Cardiol, 2012; 60: 981-987.

9. Birnie D, Tung S, Simpson C et al. Complications associated with defibrillation threshold testing: The Canadian experience. Heart Rhythm, 2008; 5: 387-390.

10. Poole JE, Johnson GW, Hellkamp AS et al. Prognostic importance of defibrillator shocks in patients with heart failure. $\mathrm{N}$ Engl J Med, 2008; 359: 1009-1017.

11. Hallas CN, Burke JL, White DG, Connelly DT. A prospective 1-year study of changes in neuropsychological functioning after implantable cardioverter-defibrillator surgery. Circ Arrhythm Electrophysiol, 2010; 3: 170-177.

12. Morgan JM, Marinskis G. Defibrillation testing at the time of implantable cardioverter defibrillator implantation: Results of the European Heart Rhythm Association survey. Europace, 2011; 13: 581-582.

13. Brignole M, Raciti G, Bongiorni MG et al. Defibrillation testing at the time of implantation of cardioverter defibrillator in the clinical practice: A nation-wide survey. Europace, 2007; 9: 540-543.

14. Healey JS, Birnie DH, Lee DS et al. Defibrillation testing at the time of ICD insertion: An analysis from the Ontario ICD Registry. J Cardiovasc Electrophysiol, 2010; 21: 1344-1348.

15. Alzueta J, Fernández JM. Spanish implantable cardioverter-defibrillator registry. Ninth Official Report of the Spanish Society of Cardiology Electrophysiology and Arrhythmias Section (2012). Rev Esp Cardiol, 2013; 66: 881-893.

16. Barold SS, Herweg B, Curtis AB. The defibrillation safety margin of patients receiving ICDs: A matter of definition. Pacing Clin Electrophysiol, 2005; 28: 881-882.

17. Jiménez-Candil J, Arenal A, García-Alberola A et al. Fast ventricular tachycardias in patients with implantable cardioverter-defibrillators: Efficacy and safety of antitachycardia pacing. A prospective and randomized study. J Am Coll Cardiol, 2005; 45: $460-461$.

18. Zeger SL, Liang KY, Albert PS. Models for longitudinal data: A generalized estimating equation approach. Biometrics, 1988; 44: 1049-1060.

19. Healey JS, Gula LJ, Birnie DH et al. A randomized-controlled pilot study comparing ICD implantation with and without intraoperative defibrillation testing in patients with heart failure and severe left ventricular dysfunction: A substudy of the RAFT trial. J Cardiovasc Electrophysiol, 2012; 23: 1313-1316.

20. Michowitz Y, Lellouche N, Contractor T et al. Defibrillation threshold testing fails to show clinical benefit during long-term follow-up of patients undergoing cardiac resynchronization therapy defibrillator implantation. Europace, 2011; 13: 683-688.

21. Bianchi S, Ricci RP, Biscione F et al. Primary prevention implantation of cardioverter defibrillator without defibrillation threshold testing: 2-year follow-up. Pacing Clin Electrophysiol, 2009; 32: 573-578.

22. Healey JS, Hohnloser SH, Glikson M et al. The rationale and design of the Shockless IMPLant Evaluation (SIMPLE) trial: A randomized, controlled trial of defibrillation testing at the time of defibrillator implantation. Am Heart J, 2012; 164: 146-152.

23. Winkle RA, Mead RH, Ruder MA, SmithNA, Buch WS, Gaudiani VA. Effect of duration of ventricular fibrillation on defibrillation efficacy in humans. Circulation, 1990; 81: 1477-1481.

24. Schoels W, Steinhaus D, Johnson WB et al. Optimizing implantable cardioverter-defibrillator treatment of rapid ventricular tachycardia: Antitachycardia pacing therapy during charging. Heart Rhythm, 2007; 4: 879-885.

25. Arias MA, Puchol A, Castellanos E. Reducing cardioverter-defibrillator shock administration by antitachycardia pacing during device charging. Rev Esp Cardiol, 2008; 61: 1087.

26. Cha Y-M, Hayes DL, Asirvatham SJ et al. Impact of shock energy and ventricular rhythm on the success of first shock therapy: The ALTITUDE first shock study. Heart Rhythm, 2013; 10: 702-708. 